\title{
Notas de historiografía agustiniana en el Perú
}

La Provincia Agustiniana del Perú no ha sido muy afortunada con sus historiadores. En el segundo tercio del siglo XVII, cuando ya había comenzado a evolucionar el concepto de historia, los Padres Maestros Anionio de la Calancha y Bernardo Torres, sus primeros cronistas, publicaron la historia del primer siglo (1551-1656), como si la historia concinuara siendo todavía el munus oratoris de las antiguas escuelas retóricas.

Era, sin duda, el P. Calancha uno de los hombres más ilustrados de su tiempo en el Virreynato. Maestro en Sagrada Teología por la Universidad de San Marcos, estaba muy versado en la Sagrada Escritura; en el púlpito habia llamado la atención desde su primera juventud: "es muy virtuoso, acepto en la predicación, aunque muy mozo", decía de él al Rey el arzobispo de Lima. Viajero sensible y alertado por todos los caminos del virreynato y religioso completāmente dedicado al estudio y a la predicación, había reunido tanto material de los archivos, de los cronistas de la Conquista y de las relaciones que le enviaron sus hermanos, que este primer tomo dedicado a historiar los años de 1551 al 1594, resultó un in-folio de casi mil páginas a doble columna ${ }^{1}$. Abundan en él digresiones de tođo género: de historia natural, prehispánica o indígena -interesantes en lo referente a las fábulas, costumbres y supersticiones de los indigenas--; de me ritísimo valor algunas para pintura del estado político, social y religioso del virreynato en el siglo XVII; no exentas de errores las dedi-

1. Antonio de la Calancha, O.S.A., Corónica Moralizada del oden de San Agustin en el Perí, con sucesos ejemplares vistos en esta Monarquia... Tomo primero (Barcelona, Imp. de Pedro Lacavallería, 1639). Hay también ejemplares de 1638 que llevan por titulo Chrónica... etc. 
cadas a la Conquista y a las guerras civiles; pero, sobre todo, moralizadoras las más, y siempre excesivas. Los árboles no dejan ver al bosque. La alusión de su contemporáneo y hermano de hábito, el Ilmo. Don Gaspar de Villarroel no es menos significativa que donosa: "Conocí a un fraile de mi Orden que con la balumba de consideraciones morales vino a ahogar la sustancia de su libro".

Al publicarse este primer tomo, el Padre Calancha recibió del Claustro de la Universidad de San Marcos el encargo de redactar la historia de la Universidad, que pedía el Rey de España para la Historia Eclesiástica de Tamayo. A ella dedicó los ocho años siguientes (1638-1646).

Continuaba reuniendo materiales para el segundo tomo de su Crónica que había concebico dividido en cinco libros. Pero, al comenzar la redacción, sintiendo que le faltaban las fuerzas y dado el gran interés que tenia en historiar la fundación del Monasterio del Prado, después de haber narrado en el primer libro la historia del Santuario de Copabana, en Bolivia, de donde era natural el P. Calancha al llegar al capítulo $\mathrm{X}$ del libro II, donde estaba narrando las fundaciones en Chile, cortó bruscamente el relato y pasó al libro quinto, dedicado al Monasterio de la Virgen del Prado. Esta es la versión del Maestro Torres, quien consideró este tomo como un paréntesis y que le ciia sencillamente como la Copacabana del Maestro Calancha. Ante la escasez de ejemplares y por no haber reparado en esta noticia de su continuador, algunos estudiosos del P. Calancha, dejándose llevar de sus fobias anti-inquisitoriales, le han inventado un proceso inquisitorial o poco menos. Recientemente todavía, el P. Avencio, desorientado por semejantes referencias, no ha reparado en afirmar que "la edición debió ser decomisada por el Tribunal del Santo Oficio o por la misma Provincia" (Los Agustinos..., p. 263). Pero la escasez de ejemplares no prueba nada; lo eran ya en 1656 los del primer tomo, por lo que el $\mathbf{P}$. Torres se vio precisado a hacer un Compendio. Los errores de la paginación y los diversos pies de imprenta confirman la versión del $P$. Torres, tal vez hasta se editaron por separado pocos ejemplares.

En cuanto continuación de la del P. Calancha, la Crónica del P. Torres comprende desde 1594 hasta fines de mayo de 1657. En 
realidad el $\mathbf{P}$. Torres no fue más que el redactor -el Sírac, dice el P. Valverde en la aprobación de la obra - del copioso material reunido por el $\mathrm{P}$. Calancha ${ }^{2}$. Así se explica que la escribiera en el breve cspacio de tres años. La sobriedad castellana del vallisoletano $P$. Torres desembarazó a la Crónica de toda extraña disquisición y exornamentación moralizaciora. Su estilo, como advierte don José de la Riva Agüero en su excelente tesis doctoral sobra la Historia en el Ferí (p. 258), "es la perfecta ancítesis del de Calancha; ajeno por completo al culteranismo y al equivoquismo, exento de toda especie ce afectación, aunque no falto de algunas arengas retóricas (para seguir sin duda el ejemplo de los historiadores clásicos), claro, llano, de excelen.e sabor castizo, de simplicidad robusta; pero lento y pesado, absolutamente desprovisto de viveza de relieve y de color".

La Continuación de la Crónica por el P. Juan Teodoro Vásquez hasia el 1721 permanece inédita todavía. En 1908, el P. Ignacio Monasierio recopiló la obra de los tres primeros Cronistas y, a base de las Actas Capitulares y otros documentos del Archivo del Convento de San Agustín, la continuó hasta 1826 en una monografía que publicó con ocasión de la reapertura al culto de la Iglesia de San Aguslin de Lima, después de la reconstrucción y transformación de principios de siglo ${ }^{3}$. En medio de sus recargadas ocupaciones como superior Mayor y como primer Director del Colegio redactó en un estilo fluido y castizo las biografías de los varones ilustres y, con una modestia que le honra a este historiador por vocación, concluía su ágil estudio monográfico con la siguiente advertencia: "La presente monografía de la Provincia Agustiniana de Perú es sólo un esbozo mal hecho del grandioso cuadro que pođrá trazar pluma más experta que la nuestra" ".

2. B. Torres, O.S.A., Crónica de la Provincia peruana del Orden de San Agustín. Lima, Imp. Julián Santos Saldaña 1,657.

Tenemos referencias de que se halla ya en prensa una segunda edición de la Crónica del P. Torres y de su Compendio del P. Calancha. Esta circunstancia actualiza la publicación de la presente nota crítica.

3. I. Monasterio, Datos para la Historia de los Agustinos en el Perú, en Recuerdo de la Inauguración del Templo de San Agustín de Lima. Imp. Moreno, Lima, 1908, pp. 133-284.

4. E.1 texto acotado, p. 284. En las pp. 254-278 publica un interesante manuscrito rotulado: SUMARIO DE LAS COSAS NOTABLES TOCANTES A RELIGION Y DE LOS VARONes ILUSTRES DE LA PROVINCIA DEL PERÙ DEL ORDEN DE LOS ERMITAÑos DE N.P. S. AGUSTIN..." Pensaba el P. Monasterio que tal vez se tratase del libro, De los Varones ilustres de la Orden, atribuido al P. Calancha 
Una carátula con la portada más representativa del barroco churrigueresco de Lima, la de la iglesia de San Agustîn, al frente, y un mapa de la Provincia Agustiniana del Perú durante la época colonial, al dorso, es ya algo incitante para iniciar la lectura del libro del P. Avencio Villarejo ${ }^{5}$, aunque no sea más que por la esperanza de hallar en él algo del grandioso cuadro que ilusionaba al P. Monasterio.

En el libro, Los Agustinos en el Perú y Bolivia, se nos ofrece la historia de la Provincia Peruana dividida en dos partes: en la primera (p. 1-362): bajo la metáfora astral del Orto, rutilante ascensión, culminación o cenit, declinación y ocaso, relata las diversas etapas históricas de la Provincia hasta el 1900, en capítulos exactamente coincidentes con la metáfora y con los siglos historiados. En la segunda (pp. 363-460), bajo el rótulo común de "Nuevo Amanecer", la actividad apostólica de los Agustinos en ambas Repúblicas desde 1900 hasta 1965.

No es del caso entrar a discutir si la división adoptada por el autor para los cinco primeros capítulos está debidamente adecuada al curso de los acontecimientos. Sabido es que toda división vertical de la historia, aunque apta para las exigencias didácticas, tiene mucho de arbitraria, porque la historia de las instituciones, como la de los individuos, es proceso continuo. Pero eso de acostarse en 1700 en la plenitud vital para amanecer en 1701 con inequívocos síntomas de decadencia en todas las manifestaciones de la vida corporativa, es ya como para que el lector se ponga alerta ante el sentido histórico que informa la narración. La de la primera parte está basada principalmente en las obras in-folio de los Cronistas, en la del P. Monasterio y en los apuntes que la constancia erudita del P. Graciano Montes fue recopilando durante varios lustros. Estos estudios, manifiesta el autor en el Prólogo, "aparte de facilitarme el trabajo, me han ahorrado meses, quizás años, de investigación".

por varios bibliógrafos desde Antonio Nicolás. En realidad se trata de una relación escrita en 1614 para enviársela al Arzobispo Meneses y conocida por ambos cronistas, como se puede ver por las notas marginales del original (Memoria de las Capellanías y Cargos Perpetuos... fols. 104-108. Pero el mismo Calancha dice claramente "Haber hallado en el archivo del convento copia de lo que se llevó al insigne arzobispo de Braga" (Crónica, p. 664 y 825).

5. A. VILlarejo, O.S.A., Los Agustinos en el Perú y Bolivia (15481965. Ed. Auxonia, Lima, 1965. 
Innecesario parece advertir que los Cronistas escribieron sus Crónicas, siguiendo el método de los analistas: referida la elección y las definiciones jurídicas del correspondiente Capitulo Provincial, pasan a narrar los acontecimientos principales que tuvieron lugar encre Capítulo y Capítulo. Los acontecimientos más frecuentes son las nuevas fundaciones y las biografías de los religiosos ilustres fallecidos durante el período. El P. Villarejo ha abandonado, en parte, este método: "Seguiremos su trayectoria a través de los Capítulos Provinciales para una mejor y más sintética visión de los acontecimientos, cronologados, no por temas, sino por fechas para establecer su ilación en el tiempo". A pesar de la precedente declaración, cada capitulo aparece dividido indefectiblemente en cuatro secciones -Gobierno y Administración, Vida religiosa, Fundaciones, Biografías- y estas secciones subdivididas a su vez en otros apartados que confieren al libro un aspecto escolar... Siete páginas de Indices Analíticos ayudan al lector a encontrar el dato buscado en este repertorio de noticias; otras cinco de Bibliografía citada o consultada, a individualizar la obra, citada con el desagradable o. c. desde la primera nota del libro; finalmente, las siglas de catorce archivos nos obligan a un buen ejercicio de la memoria, nos deslumbran y nos hacen pensar en esa "ingente cantidad de artículos, crónicas, biografías e informaciones existentes en archivos, revistas y periódicos", recopiladas, según se advierte en el Prólogo, antes del presente trabajo.

A medida que avanza la lectura, el lector comienza a sentir la impresión, cada vez más inquietante, de que esa ingente cantidad de cocumentación se vuelve anárquica al intentar encasillarla. Por la manera de citarla $y$, sobre todo, por la de interpretarla, el autor no se muestra muy familiarizado con ella. En las Visiones Panorámicas y en la sección Vida Religiosa - la más desafortunada de todas-se repiten ripiosamente las concisas y cada vez más monótonas noticias. Ya el Cronista, P. Juan Teodoro Vásquez, se lamentaba de que para las biografías había tenido que "suplir con empeños de la elocuencia la inopia de las noticias". Si esto sucedia al Cronista que escribía vidas de varones ilustres, a muchos de los cuales había alcanzado en vida, ¿qué no acontecerá a quien siglos después, con los archivos dispersos o destruidos por obra de la Revolución. se proponga escribir la historia de una institución, evadiendo la ingrata 
tarea de buscar los testimonios e interpretarlos con la más severa hermenéutica?

"Empeños de la elocuencia", vagas generalizaciones o ideas preconcebidas, nunca resultado de los hechos conocidos o testimonios aducidos, parecen también esas Visiones Panorámicas, en las que, para que la imagen del pasado encuadre en el plan prestablecicio y machaconamente repetido de ascensión, cenit, declinación y ocaso, se emplean criterios ambiguos. Así los 1.500 ó 2.000 religiosos que, dice, repletaban los conventos, los centenares de graduados en San Marcos o en San Ildefonso, y las muchas doctrinas, especialmente en Bolivia, constituyen para el autor "el cenit de la gloria" a que llegó la Provincia en el siglo XVII (p. 190). Por el contrario para el siglo XVIII, el hecho de que las clases eran dictadas principalmente por Lectores, el número extraordinario de los que solicitaban el Magisterio y la dedicación a los estudios y cátedras son sintomas y causa de decadencia, hasta en los estudios(!). ¿A qué obedece semejante disparidad de criterios?

Puestos a señalar una por una las inexactitudes de esas visiones panorámicas, tendriamos que extendernos mucho más de los límites señalados a esta nota. Veamos solamente, por via de ejemplo, las referentes al número de los religiosos y al de los graduados de lectores y Maestros, aducidas por el autor como sintomas de esplendor o de decadencia para los siglos XVII y XVIII.

En primer lugar, esos cálculos de más de 1.000 religiosos para fines del siglo XVI, y los 1.500 a 2.000 para el siglo XVII (pp. 49 y 65) en contraste con el escaso número del siglo XVIII (p. 282), resultan, por decir lo menos, poco conformes con la realidad. Por la Relación al arzobispo ĩeneses del año 1614 sabemos gue entonces los religiosos eran poco más de cuatrocientos, y que por este número y por sus 29 conventos "ha venido a aumentar la Orden de suerte que está en tan aventajado puesto como las que más lo están en este Reino" 0 .

6. Sumario de las cosas notables... etc. párrafo 4: I. Monasterio, Recuerdo de la Inauguración, p. 258. Debido a una errata de Imprenta en la enumeración de los conventos falta el de Trujillo, que figura en el original. Que la Relación haya sido escrita el año 1614 -el mismo año que vino destinado el P. Calancha desde Bolivia al Colegio de San Ildefonso consta por la conclusión del párrafo dedicado a la biografía del P. Mtr. Gabriel de Saona: "Murió a 6 de Enero, el mismo día de la Epifanía, este año de 1614". (Párrafo 7:Monasterio, p. 264). 
En esta estadística se consideraban incluidos los tres conventos de Chile -Santiago, Coquimbo, N. Sra. del Valle - y sus religiosos que, por no llegar a 80 , no habian formado Provincia independiente todavía. Relacionado este dato de 1614 con el de la Relación del Visitador P. La Raya de 1779, según la cual habia entonces 447 agustinos en 22 casas del Perú y Bolivia ? y considerando que este número de religiosos parecía excesivo a los proyectos reformistas de los golillas del Rəy Carlos III, la conclusión lógica es que, después de la gran expansión numérica $\mathrm{y}$ territorial de la segunda mitad del siglo XVI, el número de conventos y de religiosos en los siglos siguientes se mantuvo más o mencs estable. Esta es nuestra opinión, mientras nuevas evidencias no nos hagan cambiar de parecer. Aún en los tiempos de mayor dedicación a las doctrinas, el número de misioneros dedicados a la conversión de los indios fue siempre considerablemente inferior al de los que que vivian en los conventos, consagrados a la observancia y al cuidado espiritual de los negros y españoles. En el convento de Lima solamente había tantos Predicadores como Misioneros en toda la provincia ${ }^{8}$. Los Superiores vieron siempre con cierto recelo la vida solitaria en las doctrinas como poco favorecedora de la observancia religiosa. Cuando el Ilmo. P. Luis López, siendo Provincial la segunda vez (1583-87), se decidió a entregar a los señores Obispos las doctrinas de las cuatro provincias más ricas, 10 hizo para que los clérigos seculares no anduviesen desacomodados $y$, principalmente, "porque los religiosos observasen mejor su instituto en la clausura de los conventos, cumpliendo con los votos a que están obligados que no distraídos y hechos propietarios con manejo de hacienda y extrañamiento de las costumbres religiosas" ?.

7. De la Visita del P. Juan de la Raya se conservan dos ejemplares en el Archivo del Convento de San Agustín de Lima: Uno en Arm. I, c. 1., y otro en arm. II, c. $3^{\circ}$.

8. "Y hoy están ocupados (en las doctrinas) treinta y más religiosos, sin otros muchos más que predican a los españoles y negros, entresacados de cuatrocientos y más religiosos, que tiene esta Provincia. ...Y baste decir que en sólo este convento de los Reyes hay más de treinta predicadores de españoles" (Sumario, 9: MoNASTERIo, p.266). Una mano posterior -probablemente la del Mtro. Torres, a juzgar por la escrítura- añadió a este párrafo: "Hoy hay doscientos religiosos, y todos los sacerdotes son predicadores y pasan de noventa, aunque no todos prediquen por su indisposición" (Ib.). En el capítulo provincial celebrado ese mismo año, al designar de qué convento debía depender cada doctrina, se enumeran solamente veintidós doctrinas. Va. Libro II del Becerro, fol. $34 \mathrm{v}$.

9. Memorial al Virrey, citado por el Maestro Torres, quien añade es- 
Esas Universidades de San Ildefonso (intra claustra) y de San Marcos, de las que egresaban "centenares de Lectores y Maestros", se parecen más a las Universidades del siglo XX de las que egresan nutridas promociones de Licenciados y Doctores, que a las Facuitades Teológicas de los siglos XVII y XVIII. La promoción a los grados académicos en la Orden Agustiniana, y más particularmente en las Provincias de España y sus Indias durante esos siglos era mucho más compleja. Según las Constituciones entonces vigentes en la Orden, los Lectores pasaban al grado de Bachiller después de dos años de lectura o profesorado; los Bachilleres, después de otros dos, podían solicitar al Rmo. ser promovidos al Magisterio. El grado de Maestro en Sagrada Teologia no se concedía "nisi ei qui Regentis munere fungi vel publice $S$. Scriturae lectionem legere dignus atque idoneus, et integritate vitae et morum gravitate ornatus fuerit". Los Bachilleres antes del examen de magisterio y de recibir las insignias doctorales, se llemaban Licenciados o Presentados al Magisterio, al cual eran promovidos por el Prior General, si tenía potestad para ello, - Io recibian con el permiso del General "in probata aliqua Doctorum Theologorum facultate" ${ }^{10}$.

Pero, como la quinta parte de las Constituciones del 1625 referente a los estudios no se aceptó nunca en las Provincias de la Asistencia española -cuatro provincias en España y siete en las Indias-, ${ }^{11}$ la promoción al Magisterio en Sagrada Teología, término de los grados académicos en la Orden estaba regulada por constituciones apostólicas y por los decretos de los capítulos provinciales y generales, que exigian condiciones más rigurosas que las Constituciones de la Orden y limitaban el número de Maestros para cada Provincia. Así, el tiempo de profesorado para ser declarados Presenta-

te comentario: "Y la experiencia ha mostrado cuán prudente resolución fue la de aquel insigne Prelado". Crónica, p. 130.

10. Constitutiones O.E.S.A., Romae, apud Haeredem Bartholomaei Zannetti, 1625, Pars V, cap. IV, nn. 11 12.

11. En el Capítulo General celebrado en Roma el año 1679, "postulatum fuit quod capita quintae partis nostrarum Constitutionum in quibus tractatur de forma promovendis ad gradus Magisterii et Praesentatorum... mandentur servari, vel incorporentur in tertia parte, eo quod huiusmodi quinta pars in praedictis Hispaniarum et Indiarum Provinciis non fuit hucusque recepta" (AnAu., XI, 1925-26, p. 396 y 390). Cuando se publicó la siguiente edición de las Constítuciones (Romae, apud Haeredem Corbellotti, 1686) la organización de los Estudios en las Provincias españolas sirvió de modelo para otras provincias ultramontanas. 
dos era de doce años ${ }^{12}$ y nadie de las Provincias de España y sus Indias podia presentarse al examen de Doctorado en Teología en alguna Universidad, sin haber sido examinado rigurosamente y aprobado antes en la Orden por los examinadores nombrados por el Rmo. en el Capítulo General ${ }^{13}$. Después de los doce años de profesorado muchos Presentados no podian optar al grado de Maestro por ser éstos de número y no poder verificarse sin que hubiere vacante.

Para evitar los inconvenientes de la muchedumbre de Maestros y Privilegiados, la Constitución Apostólica del Papa Clemente VIII, Admonet Nos, del 13 de septiembre de 1600 limitaba y reducía a seis el número de Maestros que podian disfrutar de los privilegios magisteriales en las provincias Bética, Mejicana, de Mechoacán, del Perú de Quito y del Nuevo Reino. Estando completo el número de seis Maestros, nadie podía ser promovido al magisterio, y si lo fuere, no gozaría de las privilegios y exenciones ${ }^{14}$. El Breve de Urbano VIII, Cupientes, del 13 de mayo de 1625, facultaba al Rmo. P. General, Jerónimo de Ghettis, para conferir el Magisterio, per modum actus, a algunos religiosos que fueran hallados idóneos mediante un riguroso examen previo; pero con la restricción de que en cada Provincia de España, de las Indias y de Filipinas no se excediese el número de seis ${ }^{15}$. Los deseos de ampliar los Maestros de número en proporción al aumento de religiosos y de Lectores de las Provincias tropezaron durante muchos años con la dificultad de estas dos Constituciones Apostólicas. Cuando en el Capítulo General del 1679 se pidió insistentemente que se ampliase a doce el número de Maestros, la respuesta fue: "nihil super hac re esse innovandum ob reverentiam debitam Constituitionibus Ciementis VIII et Urbani VIII" ${ }^{16}$.

12. AnAu, XII 49.

13. Breve de Urbano VIII, Romanus Pontifex, del 25 de abril de 1625 , en Bullarium Romanum, Taurinensis ed., XIII, 320-21; L. EMPoLI, Builarium O.E.S.A., Romae, 1628 , p. 74-76. En los considerados del Breve se advierte que algunos acudian a doctorarse a las Universidades de estudios Generales, "in quibus ad gradus huiusmodi suscipiendos levisima sunt examina". En los Capítulos Generales solía nombrarse como examinadores a los tres Maestros más antiguos de la Provincia.

14. Bullarium Ordinis, p. 74-76.

15. Analecta Augustiniana XI, 61 in nota.

16. Esta fue la resolución del día 12 de junio, el 28 volvió a insistir en la petición el Definidor de Filipinas, P. 'M. Francisco Montano, y entonces "resolutum fuit quod de caetero numerus Magistrorum in Provinciis Castellae, Bethicae, Mexicanae, del Perú, Quito, Novi Regni et Mechio- 
Entretanto, la Sagrada Congregación de Obispos y Regulares urgía a la Provincia Peruana el cumplimiento de la constitución de Clemente VIII, Admonet Nos, aboliendo el grado de Presentado ex gratia y otros privilegios similares y recomendaba que en el próximo Capítulo General del 1685 se tratase de la ampliación del número de Maestros. Entonces el Capítulo decretó que en adelante en la Provincia del Perú serian doce Maestros de número, distribuyéndose nueve para los nacidos en el Perú que eran muchos más, y tres para los nacidos en España, que eran muchos menos. $Y$ este decreto fue confirmado por el Papa Inocencio IX, el 22 de enero de $1688^{17}$. Durante las dos últimas décadas del siglo XVII y las dos primeras del siglo XVIII la Provincia experimentó un rapidísimo crecimiento de religiosos y de Lectores. Las tres cuartas partes de los religiosos eran ya nativos del Perú y seguian aumentando. En las cuatro casas de estudios de la provincia había más de ciento cincuenta estudiantes de Artes y Teologia; en el Colegio de San Ildefonso enseñaban un Maestro, un Regente $y$ seis Lectores a unos ochenta estudiantes de Artes y Teología; en el del Cuzco, un Regente y cuatro Lectores a cerca de cincuenta estudiantes; en el de Chuquisaca o La Plata, cuatro Lectores a unos treinta, y en el Convento de Lima, cuyo número de alumnos se desconoce, enseñaban tres Lectores de Teología por San Marcos, tres o cuatro de los cuales eran Catedráticos de dos Lectores Jubilados de la Provincia que habian cumplido ya los doce años de profesorado, y unos veinte Doctores o Maestros en Teología por S. Marcos tres o cuatro de los cuales eran Catedráticos de la Universidad y los restantes se preparaban para las oposiciones a las posibles cátedras vacantes. Esta era a grandes rasgos la situación de los estudios en la Provincia, cuando el año 1712, solicitó a la Santa Sede elevar de doce a veinticuatro los Magisterios de número. El Papa Clemente XI por el Breve Exponi nuper del 26 de febrero de 1712 lo elevó a dieciocho ${ }^{18}$.

cani sit duodenarius hac tamen conditione, quod sex qui superaddentur accipiendi sint ex magistris supernumerariis gradu Magisterii antiquioribus... Si tamen decursu temporis forte acciderit quod nullus Magister supernumerarius existat, tunc solummodo sex magistri de numero manere debent ad praescriptum Constitutionis Clementis VIII, emanatae anno 1600 incipiente Admonet Nos. Vd. An Au. XI, 395-96.

17. AnAu., XII, 55; Breve, Pastoralis officio, en Bullarium Rom. XIX, 782-84. 
El número de religiosos continuaba aumentando, los conventos estaban bastante ricos y en 1720 se pidió ampliar el número de dieciocho a cincuenta que había aptos para el Magisterio después del illtimo examen. El 7 de octubre la Santa Sede lo amplió a veinticuatro mandando pedir el parecer del próximo Capítulo General y, después del Capítulo, celebrado en Roma junio del 1721 otro nuevo Breve, Exponi nobis, del 24 de abril de 1722 concedía otros seis magisterios para los Predicadores ${ }^{19}$, llegándose así a los treinta Maestros de número, cifra que parece no se rebasó nunca. Con la institución de estos seis magisterios para los Predicadores se daba cumplimiento a la resolución del Capítulo General de 1679 , pues hasta entonces en las Provincias Hispano-americanas sólo se promovía al Magisterio a los Lectores de Artes y Teología. A los Predicadores se les exigía, además del previo examen riguroso como para los Lectores, el haber predicado las cuaresmas de 16 años y otros muchos sermones, y ser propuestos por el Definitorio provincial al Rmo. P. General. En la Provincia del Perú quedaban veinticuatro magisterios para Ios Lectores y seis para los Predicadores.

Los Pricres Generales solian tener facultades especiales para conferir el Magisterio, intra quodlibet sexenium cincuenta Maestros supernumerarios en las Provincias y Congregaciones de Italia y cuarenta para las de fuera de Italia siempre que fueran hallados idóneos por un riguroso examen previo y reuniesen las condiciones requeridas por las Constituciones Apostólicas, de la Orden y los usos y costumbres de cada provincia. Pero los Maestros supernumerarios no tenían derecho a los privilegios y exenciones de los de número hasta que en 1726 quedó derogado el Breve Admonet Nos de Clemente VII por el Breve de Benedicto XIII, Cupientes ${ }^{20}$. Para las Provincias de fuera

18. Bullarium Romanum, XXI, 479-80.

19. IBIDEM, XXI, 848-49; 902. Parece que hay cierta hipérbole en las preces del 1722 al decir que en la Provincia habia entonces "mille et amplius religiosi necnon ex his ducenti iubilati". cuando diez años antes se dice que los lectores jubilados eran cuarenta y dos.

20. AnAu., XI, 65-66. “...utque omnibus et singulis honoribus, privilegiis, favoribus, praerrogativis... quibus alii eiusdem Ordinis in Sacra Theología Magistri etiam in publicis studiorum generalium universitatibus ad huiusmodi gradus promoti de iure, usu, consuetudine aut alias quomodolibet utuntur, potiuntur, fruuntur et gaudent"... Non obstantibus felicis recordationis Sixti V et Clementis VIII Romanorum Pontificum etiam praedecessorum nostrorum de senario Magistrorum numero...". Sixto V (1585-90) había reducido a seis los Maestros de número para la Pro- 
de Italia, tanto antes como después de esta concesión, los Superiores Generales de la Orden daban licencia a los candidatos para que, previo el examen, fueran promovidos al Doctorado o Magistero en las Universidades públicas. Así se explica que los Maestros de la Provincia Peruana lo fueran por la Universidad de San Marcos.

Por todo esto, nada más natural que en el siglo XVIII las clases fueran dictadas principalmente por Lectores, sin que ello significara un sinioma alarmante de decadencia en los estudios; así habia sido siempre en la Orden desde la época de Egidio Romano. Lo que no puede menos de extrañar es que, para describir "el cenit y 10 plenitud" del siglo XVII, se recurra a esas hipérboles: "De 1.500 a 2.000. religiosos repletaban los conventos; centenares de graduados en San Ildefonso o en San Marcos daban brillo en las Universidades púlpitos y tribunales civiles" (p. 190); que "tenian centenares de conventos y doctrinas esparcidos por todo el Reino (p. 192). Igualmente no puede menos de extrañar el que, sin reparar en la contradicción, se intente explicar la decadencia del siglo XVIII, porque "el número de los que solicitaban el magisterio era extraordinario -raro será el capítulo en que bajen de $40-$ (p. 282); y porque "la dedicación de los religiosos exclusivamente a los estudios y cátedras llenó los conventos de lectores, lectores jubilados, predicadores, maestros y doctores, todos ellos, quien más y quien menos, llenos de privilegios y exenciones" (p. 285). Todo este razonamiento carece de rigor histórico; está reclamando algunas precisiones.

En cuanto al número de religiosos, conventos y doctrinas. ya queda consignado en estas páginas lo que se dice en la Relación del 1614 enviada al arzobispo Meneses ${ }^{21}$. A mediados del siglo XVII, la Provincia tenía treinta y seis conventos priorales, unas cuarenta doctrinas y unos quinientos religiosos ${ }^{22}$. Ya entonces advertía Mal-

vincia de Castilla. Un siglo después, cuando eran ya doce los Maestros de número en la Provincia, de los doctorados en Salamanca y Alcalá solamente los cuatro más antiguos de Salamanca y los tres de Alcalá podian ser Maestros de número. Y esto no desde el momento de su Doctorado, sino después de doce años de profesorado. Vid. Decreto del Capítulo General de 1685 en $A n A u$, XII, 47 .

21. Vd. supra, nota 8.

22. JUAN MARTin Maldonado, Breue svmma (si se puede dar en lo grande) de la Provincia del Perv del Orden de Ermitaños de San Agustín, Roma, por Francisco Moneta, 1651, pp. 7-17. Juan de la Serna Maraver, 
donado en su Informe al Rmo. P. General que el convento de Lima, donde ordinariamente había unos 220 religiosos, él sólo "casi monta tanto como todo el resto y capacidad de la Provincia" ${ }^{23}$. Y el número de religiosos siguió creciendo en las últimas décadas del sigio, como se hace constar en las preces para la ampliación del número de maesiros. Los Doctores o Maestros en Teología por la Universidad de San Marcos eran nueve en 1614, veintidos en 1650, y unos veinte en 1712, cuando se elevó a dieciocho el número de Maestros de Provincia ${ }^{24}$.

$\mathbf{Y}$, por ello, se infiere que el libro del $\mathbf{P}$. Avencio Villarejo es, por supuesto, un Compendio de las Crónicas, de la obra del Paore Monasterio, de los apuntes compilados durante muchos lustros por el P. Montes y de esa historia "amplia y completa", escrita previamente por el autor. ¿Es también una síntesis histórica de la Provincia Agustiniana del Perú? En otros términos: ¿Hay en esta ingente colección de noticias la esperada concatenación de los sucesos entre sí para explicar la génesis y las consecuencias, y con los acontecimientos contemporáneos - políticos, sociales, religiosos y culturales-, en una palabra, con el ambiente político y cultural del Virreynato y de la Metrópoli, sin cuya vinculación es dificil, entender algo de una institución particular en esa época? No sé si es posible responder afirmativamente a esta pregunta. $Y$ pienso que tal incertidumbre expresa mi más importante reparo a este repertorio de noticias e ilustraciones que es el libro, Los Agustinos en el Perî $y$ Bolivia.

Están ahi la serie completa de los Capítulos Provinciales, las biografías de ochenta y tres varones ilustres $y$, lo que considero el mejor acierto, la serie de las fundaciones ilustrada con más de cien reproducciones fotográficas, en las que se nos ofrece, con ventaja sobre cualquier descripción literaria, los ambientes de conventos, claustros, iglesias, doctrinas y algunas vistas panorámicas de los

Kalendarium perpetuum... Año de 1650 (s. 1. ni nombre de impresor), en ArAg, 43 (1935) 37-38.

23. J. M. Maldonado, Breve summa, p. 9.

24. Sumario, Párrafo 12: I. Monasterio, Recuerdo, p 269. Carta del Provincial Juan de Rivera al Rmo. P. Filippo Visconti, del 28 de marzo de 1650, citada por el mismo villarejo en Los Agustinos en el Perú, p. 206. J. M. Maddonado, Breve summa, p. 8 con la advertencia referente al número senario de Maestros: "en quienes se ve claro el exceso sobre el número". 
antiguos pueblos y ciudades del Perú, donde se desarrolló o continía desarrollándose la actividad religiosa de los Agustinos. Sin embargo, uno concluye la lectura del libro con la impresión de que la obre ha sido escrita apresuradamente, que la empresa fuera más fuerte que autor, que no quedó bien ligada $y$, en definitiva, dada la ambigtiedad de criterios, muchas noticias y apreciaciones parecen sorprendentes acríticas y controvertibles.

Sorprendentes, por no mencionar más que algunas, la de que el nuevo Convento de San Agustín de Lima, emplazado a dos cuadras de la Plaza de Armas, en el cuadrilátero que trazara Pizarro, donde cada manzana queda aislada por anchas calles, "llegó a ocupar casi cuatro cuadras o manzanas" (p. 19 y 24); y que, al abandonar los Agustinos el convento viejo (1573), "se instalaron las monjas de la Trinidad, y alli estuvieron hasta que se trasladaron a su monasterio actual en 1607, porque los Agustinos vendieron en 3.000 pesos el antiguo convento al Doctor Marcos Lucio, Rector de la Universidad de San Marcos, para que se trasladase este centro de estudios desde Santo Domingo. Si bien, según Calancha, la Universidad se tralasdó de Santo Domingo a 3 de octubre de 1577..." (p. 18). ¡Cuánto desliz en estas pocas líneas! En realidad, el convento viejo fue vendido a la Universidad el 23 de diciembre de 1574, siendo Rector Juan de Herrera, y allí se trasladó entonces la Universidad desde Santo Domingo. El Dr. Marcos de Lucio, que firma el contrato como fiador, dijo al día siguiente en el Cabildo de Lima "que la dicha Universidad tenía comprada la casa que fue de sancto Agustín junto a san Marcelo, y que se ha de pagar de aquí a seis meses, tomada posesión de ella y hecho el claustro en que está acordado que se lea desde Pascua de los Reyes, cánones y Leyes, y Teología y Artes, y Filosofía y la Esfera" 25. Era Rector dos años después, cuando a petición suya lo mandó vender el Virrey don Francisco de Toledo, para trasladar nuevamente la Universidad, el 3 de octubre de 1576, a 10 que actualmente es el Palacio Legislativo ${ }^{26}$.

25. Cabildos de Lima, Libro VII, sesión 24-XII-1574. El documento de venta puede verse en Luis A. Eguiguren, Alma Mater, p. 291.

26. Para lo referente a los periodos rectorales en San Marcos, vd. Eguiguren, Diccionario, hist. cronol., pp. 87, 120 y 124. A este traslado de la Universidad al Palacio Legislativo se refiere el P. Calancha en la Crónica (p. 690), donde parece desconocer el traslado anterior al antiguo con- 
Ya desde la Dedicatoria (se repite después en las pp. 182 y 206) no puede menos de extrañar esa forma afrancesada de "Philipe de Bisconde" en que se transforma el nombre y apellido del milanés Rmo. P. Filipo Visconti.

Acrítico completamente, como en Calancha, nos parece eso de atribuir a un milagro la muerte del Maestro Fray Luis de León a los pocos días de haber sido elegido Provincial, sólo para que viniera a Lima la copia del Cristo de Burgos; a lo que se añade el suponerle ya Provincial antes del Capítulo: "Fueron interceptados por un emisario mandado por el Provincial de Castilla Fray Luis de León" (p. 20).

\section{Quirino Fernandez.}

vento de San Agustín. Pero años después, el mismo Calancha, mejor documentado en los Archivos de la Universidad, no puede ser más explícito: "escogiose por eminente sitio para fundar los estudios y la Universidad (al salir de Santo Domingo), el que dejaba entonces la Orden de San Agustín, a seis cuadras de la plaza, donde después se fundó el monasterio de monjas de la Santisima Trinidad y la parroquia de San Marcelo". Vd. Relación Histórica de la Universidad de San Márcos por el P. Mtro. Antonio de la Calancha, reimpresa por Eguigures en su Dicc. hist. cronol., pp. 1-30. El párrafo acotado se encuentra en la p. 4. 